\title{
Reliability Analysis of Facility Systems Subject to Edge Failures: Based on the Uncapacitated Fixed-Charge Location Problem
}

\author{
Zongtian Wei ${ }^{1,2^{*}}$, Huayong Xiao ${ }^{1}$ \\ ${ }^{1}$ Department of Applied Mathematics, Northwestern Polytechnical University, Xi'an, China \\ ${ }^{2}$ Department of Mathematics, Xi' an University of Architecture and Technology, Xi' an, China

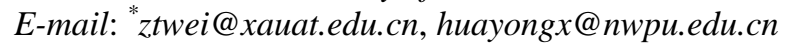 \\ Received August 11, 2011; revised Sepetember 19, 2011; accepted September 30, 2011
}

\begin{abstract}
A facility system can be modeled by a connected graph in which the vertices represent entities such as suppliers, distribution centers or customers and the edges represent facilities such as the paths of goods or information. The efficiency, and hence the reliability, of a facility system is to a large degree adversely affected by the edge failures in the network. Such failures may be caused by various natural disasters or terrorist attacks. In this paper, we consider facility systems' reliability analysis based on the classical uncapacitated fixed-charge location problem when subject to edge failures. For an existing facility system, we formulate two models based on deterministic case and stochastic case to measure the loss in efficiency due to edge failures and give computational results and reliability envelopes for a specific example.
\end{abstract}

Keywords: Facility System, Reliability, Edge Failure, Uncapacitated Fixed-Charge Location Problem

\section{Introduction}

It is well known that facility location decisions are strategic in a supply chain, or more generally speaking, in a facility system design, since facility location decisions are costly and difficult to reverse, and their impact spans a long time horizon.

We use the term "facility" here in its broadest sense. That is, it is meant to include facilities such as factories, warehouses, retail outlets, schools, hospitals, and satellites, as well as transportation lines, cables to name but a few that have been analyzed in the research literature.

Every facility system in operation maybe faces various disruptions. Such disruptions have begun to receive significant attention from practitioners and researchers after the terrorist attacks of September 11, 2001. Facility system disruptions can have significant physical costs (e.g., damage to facilities, inventory, electronic networks, and infrastructure) and subsequent losses due to downtime. A recent study [1] estimates the cost of downtime (in terms of lost revenue) for several online industries that cannot function if their computers are down.

We view the structure of a facility system as a connected graph in which the vertices represent facilities such as subway stations, distribution centers, etc., and the edges represent facilities such as the paths of goods (e.g., transportation lines) or information (e.g., cables). In this paper, we distinguish two kinds of facilities, e.g., "vertex facilities" and "edge facilities". For simplicity, we call them "vertices" and "edges", respectively.

It is well known that, regardless of intentional strikes or natural disasters, edges are easily to be damaged. In most cases, facility system disruptions are caused by the failures of edges, e.g., closure of highway because of the inclement weather, traffic jam, road damage caused by earthquakes or debris flows.

In this paper, adopting the facility location analysis framework, we will mainly consider facility systems' reliability analysis based on the classical uncapacitated fixed-charge location problem (UFLP) when subject to edge failures, whereby we consider an existing facility system in which the facilities may or may not be located optimally. The edges may be lost due to natural disasters or terrorist attacks. We want to know the efficiency of the remaining system under such circumstances. We will formulate two models based on deterministic case and stochastic case to measure the loss in efficiency due to edge failures and give computational results and reliabil- 
ity envelopes for a specific example.

The remainder of the paper is organized as follows. In Section 2 we review some related literature. In Section 3, we formulate two reliability models based on the UFLP and edge failures. We use a scenario-based algorithm to compute a specific example and give the results and reliability envelopes in Section 4. Section 5 is a summary of this paper.

In the following, by "loss" we refer to the edge disruptions (failures) mentioned above or, sometimes the necessary closure.

\section{Literature Review}

In this section, we briefly review the facility systems' reliability under disruptions.

The concept of facility system reliability is related to network reliability theory, which is concerned with calculating or maximizing the probability that a graph remains connected after random failures due to congestion, disruptions, or blockages. Typically, this literature considers disruptions to the links of a network, but some papers consider node failures [2], and in some cases the two are equivalent. Given the difficulty in computing the reliability of a given network, the goal is often to find the minimum-cost network with some desirable properties like 2-connectivity [3,4], k-connectivity [5], or special ring structures [6].

The reliability of a facility system is the probability that all suppliers are operable [7]. Generally speaking, the key difference between networks reliability and facility systems reliability is that the former are primarily concerned with connectivity; they consider the cost of constructing the network but not the cost that results from a disruption, whereas the latter consider both types of costs and generally assume connectivity after a disruption [8].

The facility location problem is a classical, combinatorial optimization problem to determine the number and locations of a set of facilities and assign customers to these in such a way that the total cost is minimized. Two types of costs are considered in the problem. A setup cost (facility cost) occurs while a facility is opened, and a connection cost occurs while a customer is assigned to the opened facility.

If an arbitrary number of customers can be connected to a facility, the problem is called uncapacitated facility location problem (UFLP) [9]. The UFLP is NP-hard [10] and have been extensively studied. Lots of algorithms, exact and heuristic, have been developed in the past decades [1,11-13].

A number of papers in the location literature have addressed the problem of finding the optimal location of protection devices to reduce the impact of possible disruptions to infrastructure systems.

For example, Carr et al. [14] presents a model for optimizing the placement of sensors in water supply networks to detect maliciously injected contaminants. James and Salhi [1] investigate the problem of placing protection devices in electrical supply networks to reduce the amount of outage time.

Church et al. [15] presented a model called the r-interdiction median problem. This model can be used to identify which $r$ of the existing set of p-facilities, when interdicted or lost impacts delivery efficiency the most. Such a model can be used to identify the worst case of loss, when losing a pre-specified number of facilities. The model is restricted in two ways: it is based upon the assumption that the terrorist or interdictor is successful in each and every strike, and it is also based upon the assumption that exactly $r$ facilities will be struck and lost. Such a model does address a worst case scenario, but it does not exactly capture the issues that would be key to understanding the range of failures and possible outcomes.

In [16], the authors argued that first, it is important to recognize that a strike or disaster may not impair a facility's operation. That is, a terrorist strike may be successful only a certain percentage of the time. The same is true for a natural disaster. When it does occur, there is a threat that operations at a facility may need to be suspended, but it is not absolute e. Second, interdiction may not be intelligent when the strikes impact a non critical facility. Although it is important to model "worst-case" scenarios, it is also important to model and understand the range of possible failures and impacts. Therefore, they proposed a family of models which can be used to model the range of possible impacts associated with the threat of losing one or more facilities to a natural disaster or intentional strike. They show how to model deterministic loss and probabilistic loss. In addition, they presented results associated with the application of the worst case and the best case expected loss models to a data set.

There is a mature literature on reliable network (e.g., supply chain network (SCN)) design and analysis under component failures. Unfortunately, so far we have not found the explicit study of facility systems' reliability subject to edge failures. In fact, the reliability, and hence the efficiency, of a facility systems is to a large degree adversely affected by failures of the edges. Such failures may be caused by congestion, inclement weather, earthquakes, debris flows, sandstorms, strikes or terrorist attacks. Thus the network based facility system reliability models we will study are more practical and closer to the 
reality of facility system management.

\section{Facility System Reliability Analysis Models}

In this paper, we use the total operational cost as the efficiency measure of a facility system. The notion reliability is defined to be the ratio of the system's efficiency and the efficiency after some edges have failed. We distinguish two cases: deterministic and stochastic, to formulate the UFLP-based reliability analysis models that evaluate the efficiency of a facility system after some edges have failed.

Suppose that we have a system of some operating facilities supplying a set of demand points. If each facility can serve any assigned demand, then we can assign each customer to their closest facility (as measured by cost or distance). We can define weighted distance for a demand-facility interaction as the distance from the demand to their closest facility weighted by the number of trips needed to supply that demand from a facility utilizing some type of transport mode (e.g., truck). Thus, we can measure the overall efficiency of the system as the total truck-miles of travel needed to supply all of the demand from the set of located facilities.

The exact opposite of the UFLP occurs when we consider an existing system in which the facilities may or may not be located optimaally.

When either closing or considering the loss of one or more edges by a disaster, the basic question is what happens to the operating efficiency of the system. We can measure this loss of efficiency by calculating the resulting increase in delivery cost (or loss of the system efficiency) as a reliability envelope. The details will be discussed in Section 4.

The following are the notations for our formulations.

Sets

I: set of customers, indexed by $i$.

$J$ : set of potential facility locations, indexed by $j$.

Parameters

$h_{j}$ : demand at customer $i \in I$.

$f_{j}$ : fixed cost of open a facility at $j \in J$.

$c_{e}$ : "delivery" cost per unit per "length" through road $e$.

$\alpha$ : weight of the fixed cost in the objective function.

We view a facility system as a weighted connected simple graph $G(V, E, H, D)$, where $V=I \cup J$; $E$ is the edge set with the edges denoting goods or information paths; $H$ is the vertex weight set with the weight $h_{i j}$ of vertex $i$, denoting the demand of customer $i$, and $D$ is the edge weight set with the weight $d_{i j}$ of edge $(i, j)$, denoting the length (e.g., distance) between $i$ and $j$ under the existing conditions. By $d_{i j}$ we also denote the shortest path length (or distance) between $i$ and $j$ if $(i, j) \notin E$.
Assume that $X$ is an feasible solution of the UFLP, i.e., $X_{j}=1$, if a facility is established at location $j \in J ; 0$, otherwise. Let $C$ be the opened facility (server) set corresponding to $X$, and $F \subseteq E$ be the potential failure edge set, where the edge failure is defined as an edge losses its designed function completely. Therefore, a failed edge in $G$ is equivalent to delete (or close) the corresponding line from the facility system. We also assume that edge failures are independent and multiple edge failures may occur simultaneously.

\subsection{The Deterministic Reliability Models}

Let $S_{r}$ be the set of scenarios corresponding to the closure or deletion of $r$ edges from $G$, i.e., every $s \in S_{r}$ explicitly specifies the failed $r$ edges in $F$. Let $d_{i j s}$ be the shortest distance between customer $i$ and facility $j$ in scenario s. Define $N_{i s}=\left\{j \in C: d_{i j s}<+\infty\right\}$. Assume that in any scenario $s \in S_{r}$, customer $i$ is served by an opened facility $j \in C$ which is the nearest one from $i$ if $N_{i s} \neq \phi$. Associated with each customer $i$ is a per-unit penalty cost $\theta_{i}$ that represents the cost of not serving the customer if $N_{i s}=\phi . \theta_{i}$ may represent a lost-sales cost, or the cost to pay a competitor to serve the customer temporarily. We define the assignment variables as $Y_{i j s}=1$, if customer $i$ is served by facility $j$ in scenario $s$; 0 , otherwise. Assume that in any scenario $s \in S_{r}$, customer $i$ is served by an opened facility $j \in C$ which is the nearest one from $i$.

We formulate the deterministic reliability model (DRM) as the following integer-linear programming problem:

$$
\begin{gathered}
\min _{s \in S_{r}}\left(\alpha \sum_{j \in C} f_{j} X_{j}+(1-\alpha)\left(\sum_{i \in I, N_{i s} \neq \varphi} \sum_{j \in C} c h_{i} d_{i j s} Y_{i j s}+\sum_{i \in I, N_{i s}=\varphi} h_{i} \theta_{i}\right)\right) \\
\text { s.t. } \sum_{j \in C} Y_{i j s} \leq 1, \forall i \in I, s \in S_{r} \\
Y_{i j s} \in\{0,1\}, \forall i \in I, \quad j \in C, s \in S_{r}
\end{gathered}
$$

For a given edge loss level $r$ (the number of closed or deleted edges), this model can be used to evaluate a facility system's operational efficiency under the best case, namely the minimal loss of the system's efficiency.

Changing "min" to "max" in the objective function, then we obtain the worst case model, that is the model to measure the maximal increase in weighted distance under the edge failure level $r$.

\subsection{The Stochastic Reliability Models}

The reliability model formulated above is based upon a deterministic analysis. Up to this point we have modeled edge loss a certainty. We now consider the case where 
loss is not a certainty upon an edge failure. Usually, the chances of losing an edge are based upon some probability. We wish to derive the maximal or minimal expected efficiencies associated with an existing system. To do this we need to identify both the worst case and the best case expected outcomes.

Let $F \subseteq E$ be the target edge (the potential failure edge) set of an attack. Assume that an attacker can hit each edge in $F$ at most once and that the edges in $F$ will be hit simultaneously.

Let $S_{r}$ be the scenario set when $r(0 \leq r \leq|F|)$ edges in $F$ have been attacked. Each $s \in S_{r}$ specifies which $r$ edges in $F$ have been attacked. Define

$$
E_{s}=\{e \in F \mid e \text { is attacked in scenario } s\},
$$

then any $F_{s} \subseteq E_{s}$ can be used to represent a failed edge set in scenario $s$, so we call $F_{s}$ the sub-scenario of $s$. Let $j$ be a nearest opened facility to customer $i$ and $d_{i j F_{s}}$ be the shortest distance between them in scenario $F_{s}$. Define $N_{i F_{s}}=\left\{j \in C: d_{i j F_{s}}<+\infty\right\}$. Assume that in any scenario $F_{s}$, customer $i$ is served by an opened facility $j \in C$ which is the nearest one from $i$ if $N_{i F_{\mathrm{s}}} \neq \phi$. Let $p_{j}$ be the failure probability of edge $j \in F$ after one attack. It is easy to see that scenario $F_{s}$ occurs with probability

$$
p_{F_{s}}=\prod_{j \in F_{s}} p_{j} \prod_{j \in E_{s} \backslash F_{s}}\left(1-p_{j}\right)
$$

Denote the assignment variables as $Y_{i j F_{s}}=1$, if customer $i$ is served by an open facility $j$ in scenario $F_{s} ; 0$, otherwise.

Let $\mathrm{C}$ be the opened facility (server) set of an existing facility system. We formulate the stochastic reliability model (SRM) as following (The penalty cost $\theta_{i}$ are defined in Section 3.1.):

$$
\begin{aligned}
& \min _{s \in S_{r}}\left(\alpha \sum_{j \in C} f_{j} X_{j}+(1-\alpha)\right. \\
& \left.\times\left(\sum_{F_{s} \subseteq E_{s}} p_{F_{s}} \sum_{i \in I, N_{i F_{s}} \neq \varphi} \sum_{j \in C} c h_{i} d_{i j F_{s}} Y_{i j F_{s}}+\sum_{i \in I, N_{i F_{s}}=\varphi} h_{i} \theta_{i}\right)\right) \\
& \text { s.t. } \sum_{j \in C} Y_{i j F_{s}}=1, \forall i \in I, F_{s} \subseteq E_{s} \\
& Y_{i j F_{s}} \in\{0,1\}, \forall i \in I, j \in C, F_{s} \subseteq E_{s}
\end{aligned}
$$

The objective function selects $r$ edges from $F$ to minimize the weighted distance expectation after $r$ edges in $F$ have been attacked. Constraints (3) require that each customer be served by at most one server in any scenario $F_{s}$. Constraints (4) require the assignment variables to be binary.

For a given failure level $r$, this model can be used to evaluate a facility system's operational efficiency under the best case. Changing "min" in the objective function to "max", then we obtain the worst case model, that is the model to measure the maximal increase in expected weighted distance under failure level $r$.

\section{The Reliability Envelopes}

The models described above can be applied to a given facility system over a range of edge loss level $r$. We use the weighted distance to measure the efficiency of a facility system and efficiency is measured at $100 \%$ if all edges are operating. If an edge is lost due to a natural disaster, intentional strike or planned closure, then the efficiency is lost and overall efficiency decreases. If many edges exist, then there exist several possible outcomes of losing just one edge. One can easily enumerate each of the possible ways of losing one edge as well as calculate the impact of each possible loss in terms of changes in efficiency. The results of this series of calculations will define a range of losses from the best case (i.e. the least decrease in efficiency) to the worst case (i.e. the greatest decrease in efficiency). We then have a region defined by an upper curve and a lower curve, where the upper and the lower curve represent the solutions of the least or the greatest impact associated with a given loss level, respectively. The region depicted between these two curves can be defined as the operational envelope or reliability envelope. For a given edge loss level, this envelope specifies the range of possible system performance from the best-case to the worst-case. Actual performance will fall within this range.

In this section we apply the DRM and the SRM to a data set to generate reliability envelopes. Our data set is derived from the 2008 China census data: a 49-node set consisting of the capitals of all the provinces in China plus the two special administrative regions Hong Kong and Macau, as well as other 15 big cities. The demand of city $i, h_{i}$ is settled to be the city's administrative region population divided by 10000 . The transportation links (edges) are set to the recent national highways and the transportation costs per unit per length through different roads are all set to $c=0.005$. The fixed cost of facilities setup are estimated by considering the factors such as local labor price, facility size, and other natural conditions.

By using the above data set we optimally solve an UFLP with $\alpha=0.7$ in order to site an existing facility system. Figure 1 shows the optimal solution, where the 8 distribution centers are city 4 , city 7 , city 11 , city 20 , city 24, city 26, city 28 and city 45, and the edges marked by red color represent the delivery routes from each distribution center to its customers. 
Given this operating system of 8 facilities and a potential failure edges set which is consisted of 8 edges: $\mathrm{F}=$ \{1 (5,47), $2(3,4), 3(7,40), 4(10,11), 5(28,31), 6(24,25)$, $7(17,45), 8(21,49)\}$ (Notice that the sub-graph $G-F$ is connected, i.e., both of $N_{i s}$ and $N_{i F_{s}}$ are not $\phi$ ), We solve the worst-case DRM and the best-case DRM. The solutions are given in Table $\mathbf{1}$.

In Table 1, for each edge loss level, the objective function values and efficiency for each case are also given as a percentage, where $100 \%$ represents the operating level before edge failures.

Obviously, the edges shown in volume 6 of Table 1 are the most important objective of protection.

Figure 2 presents the values of operational efficiencies (in percent) as a graph, depicting the lower and the upper boundaries of the reliability envelope. Notice that the greatest marginal impact for the worst case occurs when the edge loss level is small while for the best case occurs when the edge loss level is great.

It is also important to note that the greatest difference between the worst case and the best case of the envelope occurs when the edge loss level is moderate.

By using the same data set as in the above DRM, we then solve the SRM with edge failure probability $\mathrm{p}=0.3$ and $p=0.7$, respectively. The solutions of the latter and the corresponding reliability envelope are showed in Table 2 and Figure 3, respectively.

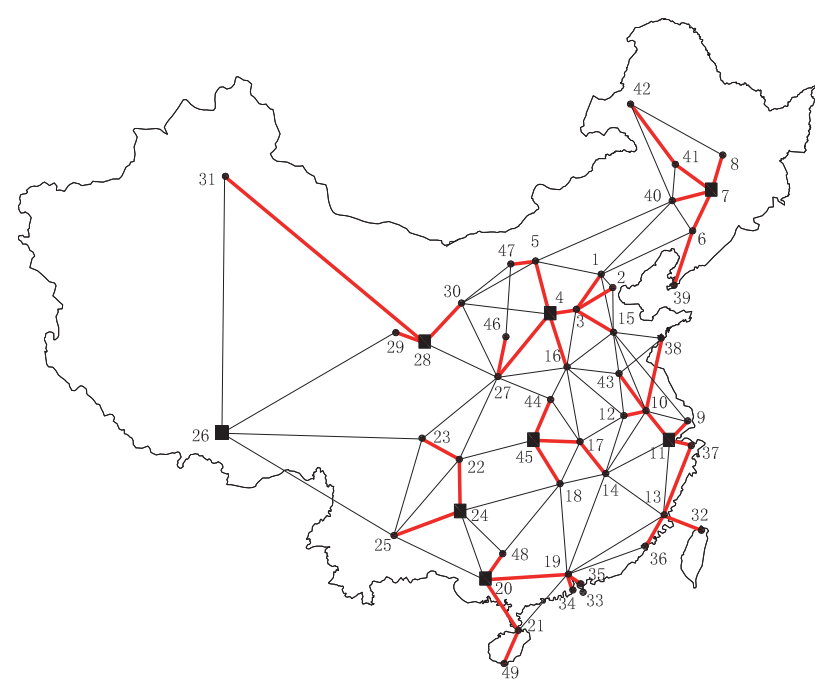

Figure 1. Optimal solution of a UFLP with $\alpha=0.7$.

Table 1. Results of the Worst-case DRM and the Best-Case DRM with $\alpha=0.7$.

\begin{tabular}{ccccccc}
\hline Level & \multicolumn{3}{c}{ Best-Case } & \multicolumn{3}{c}{ Worst-Case } \\
\hline$r$ & Objec. Value & Failed Edges & Efficiency & Objec. Value & Failed Edges & Efficiency \\
\hline 0 & $31,890.22$ & - & $100 \%$ & $31,890.22$ & - & $100 \%$ \\
1 & $31,995.78$ & 3 & $99.67 \%$ & $40,117.94$ & 8 & $79.49 \%$ \\
2 & $32,105.80$ & 1,3 & $99.33 \%$ & $43,361.45$ & 2,8 & $73.55 \%$ \\
3 & $32,241.20$ & $1,3,6$ & $98.91 \%$ & $44,169.38$ & $2,4,8$ & $72.20 \%$ \\
4 & $32,484.10$ & $1,3,5,6$ & $98.17 \%$ & $44,813.33$ & $2,4,7,8$ & $71.16 \%$ \\
5 & $33,079.71$ & $1,3,4,5,6$ & $96.40 \%$ & $45,056.22$ & $2,4,5,7,8$ & $70.78 \%$ \\
6 & $33,723.66$ & $1,3,4,5,6,7$ & $94.56 \%$ & $45,191.63$ & $2,4,5,6,7,8$ & $70.57 \%$ \\
7 & $37,179.48$ & $1,2,3,4,5,6,7$ & $85.77 \%$ & $45,301.65$ & $1,2,4,5,6,7,8$ & $70.40 \%$ \\
8 & $45,407.21$ & $1,2,3,4,5,6,7,8$ & $70.23 \%$ & $45,407.21$ & $1,2,3,4,5,6,7,8$ & $70.23 \%$ \\
\hline
\end{tabular}

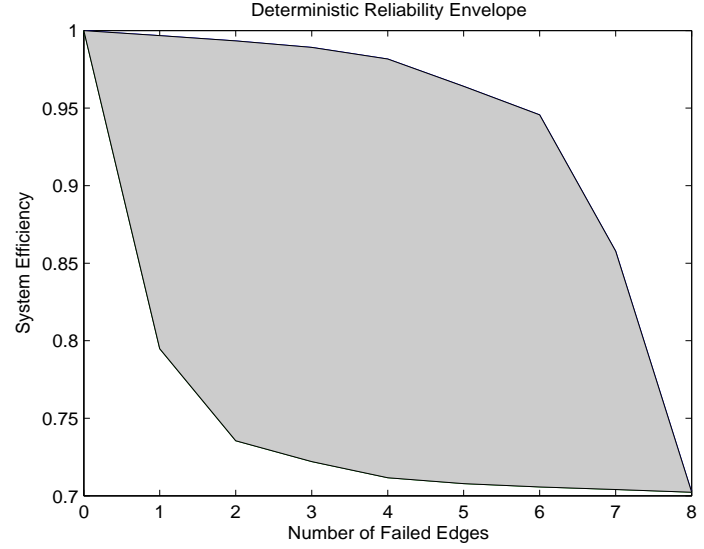

Figure 2. Reliability envelope of solutions in Table 1.

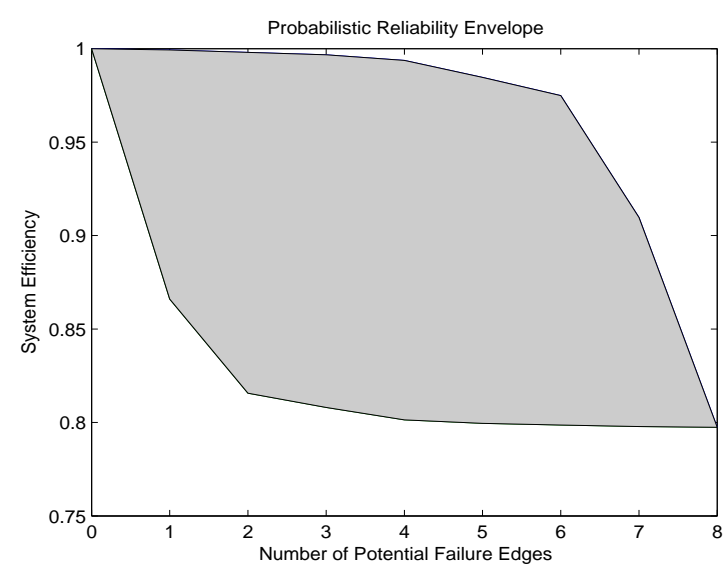

Figure 3. Reliability envelope of solutions in Table 2. 
Notice that the characteristics of this reliability envelope are similar to that of the DRM except that, on the same edge loss level, the efficiency losses of the SRM are less than that of the DRM, since we assume that the failure probability of an edge under a strike is 1 in the DRM. We can also observe from Table 2 that, for a given edge attacked level, the most important edges of protection are shown in volume 6 .

\section{Summary and Conclusions}

In this paper, we propose two types of scenario based facility location models in order to analyze the reliability of an existing facility system when subject to edge failures. We distinguish deterministic and stochastic cases to formulate and compute a specific example. Reliability envelopes in these two different cases are also given. The information in the reliability envelopes can be very useful in looking at ways to protect a facility system. Whether the protection is against a natural disaster or intentional strike, reducing the probability of success even by modest amounts could have an impact on system efficiency. For example, this could be done by placing extra strength in key sections such as bridges or tunnels which spaced in disaster-prone areas, or by adding a surveillance system with guards to help protect against an intruder. Either techniques may not completely eliminate a loss, by reduce the edge failure probability to zero, but such strategies may generate more benefits in terms of improved expected system operating efficiencies than what it might cost. Therefore, the value of our analysis could lead to higher levels of safety as well as efficient levels of resource allocation for security measures (whether that involves a possible natural disaster or an attacker).

While a large body of literature focus on the reliable and robust facility system design and analysis under component failures, the existing works are mainly concentrated on the "node" (e.g., suppliers, distribution centers) losses. To the best of our knowledge, researchers and practitioners have not paid enough attention to the impact of edge failures to the efficiency of a facility system by so far. Comparing to the related works done in this field, our work have at least the following innovations.

Firstly, combining edge failures into facility systems reliability analysis is more realistic than only considering vertex failures. In fact, natural disasters or intentional attacks damage the edges of a facility system more easily. Secondly, in the PMP and other uncapacitated facility location problems, when one or more "nodes" have failed, the overall supplement of the facility system will decrease dramatically but the total demand does not change. If in this situation all demands must to be met, then every node must has no any capacity limit. However, the capacity of nodes are designed a priori, when a vertex failure happen, how can it's adjacent nodes guarantee the increased demands, let alone more than one vertex failure occur simultaneously?

The recovery time for a failed edge maybe shorter than that for a failed vertex, but this is not always the case. So we do not explicitly point out the time horizon in our models. In addition, the evaluation of edge failure probability is important and difficult, and we will discuss this question in another work. We set the failure probability of all edges as the same when solving the example since we aimed to demonstrate the impact of edge failures to a facility system efficiency. Naturally, we need to consider the further research directions as follows.

We assume that the edge failures are independent each other, but in practice, once an edge failed, the function of its adjacent edges will be impacted. Modeling the reliable facility systems and related problems under this situation are worthy of study.

Table 2. Results of the worst case SRM and the best case SRM with edge failure probability 0.7 .

\begin{tabular}{|c|c|c|c|c|c|c|}
\hline \multirow{2}{*}{$\begin{array}{c}\text { level } \\
r\end{array}$} & \multicolumn{3}{|c|}{ Best-Case } & \multicolumn{3}{|c|}{ Worst-Case } \\
\hline & Objec. Value & Attacked Edges & Efficiency & Objec. Value & Attacked Edges & Efficiency \\
\hline 0 & $31,890.22$ & - & $100 \%$ & $31,890.22$ & - & $100 \%$ \\
\hline 1 & $31,912.22$ & 1 & $99.93 \%$ & $36,826.85$ & 8 & $86.60 \%$ \\
\hline 2 & $31,952.84$ & 1,6 & $99.80 \%$ & $39,097.31$ & 2,8 & $81.57 \%$ \\
\hline 3 & $31,995.07$ & $1,3,6$ & $99.67 \%$ & $39,469.42$ & $2,4,8$ & $80.80 \%$ \\
\hline 4 & $32,092.22$ & $1,3,5,6$ & $99.37 \%$ & $39,791.40$ & $2,4,7,8$ & $80.14 \%$ \\
\hline 5 & $32,390.03$ & $1,3,4,5,6$ & $98.46 \%$ & $39,888.56$ & $2,4,5,7,8$ & $79.95 \%$ \\
\hline 6 & $32,712.01$ & $1,3,4,5,6,7$ & $97.49 \%$ & $39,930.78$ & $2,3,4,5,7,8$ & $79.86 \%$ \\
\hline 7 & $35,056.77$ & $1,2,3,4,5,6,7$ & $90.97 \%$ & $39,971.40$ & $2,3,4,5,6,7,8$ & $79.78 \%$ \\
\hline 8 & $39,993.41$ & $1,2,3,4,5,6,7,8$ & $79.74 \%$ & $39,993.41$ & $1,2,3,4,5,6,7,8$ & $79.74 \%$ \\
\hline
\end{tabular}


We only consider the analysis of an existing facility system in this paper. Although the impact of edge failures to a facility system is less than that of vertex failures, especially when the scale of a facility system is large, the design of a reliable/robust facility system considering edge failures is also an important problem. We will also study the facility system reliability problems considering both edge failures and vertex failures in the future.

We do not consider the edge capacity constraint in our models, so it is obvious that not every edge failure will change the objective function value. For example, the potential failure edges are all in the delivering paths of the optimal solution shown in Figure 1. Despite these potential failure edges are selected according to reality, thre exist other potential failure edges. Due to the failure probability of these edges are relatively small and the consideration of computational time, we omit them. When consider the capacity of edges, the case will be different. Modeling and algorithm of problems in this case may be interacted.

Finally, our models are based on a classical facility location problem, the UFLP. Next, we will consider edge failures in more extensive reliable facility location problems, e.g., CFLP (see [11]) etc.

\section{Acknowledgements}

This paper was supported by the Open Fund of Xi'an Jiaotong University (No.2010-4), the SXESF (No. 09JK 545) and the BSF (No.JC0924).

\section{References}

[1] J. C. James and S. A. Salhi, "Tabu Search Heuristic for the Location of Multi-Type Protection Devices on Electrical Supply Tree Networks," Journal of Combinatorial Optimization, Vol. 6, No. 1, 2002, pp. 81-98. doi:10.1023/A:1013322309009

[2] H. A. Eiselt, M. Gendreau and G. Laporte, "Location of Facilities on a Network Subject to a Single-Edge Failure,” Networks, Vol. 22, No. 3, 1992, pp. 231-246. doi:10.1002/net.3230220303

[3] C. L. Monma, "Minimum-Weight Two Connected Spanning Networks," Mathematical Programming, Vol. 46, No. 2, 1990, pp. 153-171. doi:10.1007/BF01585735
[4] C. L. Monma and D. F. Shalcross, "Methods for Designing Communications Networks with Certain 2-Connected Survivability Constraints,” Operations Research, Vol. 37, No. 4, 1989, pp. 531-541. doi:10.1287/opre.37.4.531

[5] D. E. Bienstock, E. F. Brickell and C. L. Monma, "On the Structure of Minimum Weight Kconnected Spanning Networks," SIAM Journal on Discrete Mathematics, Vol. 3, No. 3, 1990, pp. 320-329. doi:10.1137/0403027

[6] B. Fortz and M. Labbe, "Polyhedral Results for TwoConnected Networks with Bounded Rings," Mathematical Programming Series A, Vol. 93, No. 1, 2002, pp. $27-$ 54. doi:10.1007/s10107-002-0299-9

[7] M. Bundschuh, D. Klabjan and D. L. Thurston, "Modeling Robust and Reliable Supply Chains,” Working Paper, University of Illinois, Urbana-Champaign, IL. 2003.

[8] D. A. Erlenkotter, "A Dual-Based Procedure for Uncapacitated Facility Location,” Operations Research, Vol. 26, No. 6, 1978, pp, 992-1009. doi:10.1287/opre.26.6.992

[9] M. L. Balinski, "Integer Programming: Methods, Uses, Computation,” Management Science, Vol. 12, No. 3, 1965, pp. 253-313. doi:10.1287/mnsc.12.3.253

[10] R. D. Galvao and L. A. Raggi, "A Method for Solving to Optimality Un-Capacitated Location Problems,” Annals of Operations Research, Vol. 18, No. 1, 1989, pp. 225-244. doi:10.1007/BF02097805

[11] L. V. Snyder and Z. J. M. Shen, "Managing Disruptions to Supply Chains," The Bridge National Academy of Enginee-Ring, Vol. 36, 2006, pp. 39-45 (Forthcoming).

[12] P. B. Mirchandani and R. L. Francis, "Discrete location Theory,” Wiley, New York, 1990.

[13] J. Krarup and P. M. Pruzan, "The Simple Plant Location Problem: Survey and Synthesis," European Journal of Operational Research, Vol. 12, No. 1, 1983, pp. 36-81. doi:10.1016/0377-2217(83)90181-9

[14] R. D. Carr, et al. "Robust Optimization of Contaminant Sensor Placement for Community Water Systems," Mathematical Programming, Vol. 107, No.1-2, 2005. 337-356. doi:10.1007/s10107-005-0689-X

[15] R. L.Church, M. P. Scaparra and R. S. Middleton, "Identifying Critical Infrastructure, the Median and Covering Facility Interdiction Problems," Annals of the Association of American Geographers, Vol. 94, No. 3, 2004, pp. 491502. doi:10.1111/j.1467-8306.2004.00410.x

[16] R. L. Church and M. P. Scaparra, "Critical Infrastructure,” Chapter 11, Springer Berlin Heidelberg, Berlin, 2007. 